\title{
Factors associated with the whole foods consumption by adults and elderly
}

\author{
Fatores associados ao consumo de alimentos integrais por adultos e idosos \\ Factores asociados al consumo de alimentos integrales por adultos y ancianos
}

Received: 08/25/2021 | Reviewed: 09/03/2021 | Accept: 09/13/2021 | Published: 09/14/2021

\author{
Patricia Thomazi \\ ORCID: https://orcid.org/0000-0002-9351-518X \\ Federal University of Fronteira Sul, Brazil \\ E-mail: patriciathomazi@ hotmail.com \\ Camila Elizandra Rossi \\ ORCID: https://orcid.org/0000-0003-0925-0703 \\ Federal University of Fronteira Sul, Brazil \\ E-mail: camilarossi@uffs.edu.br \\ Eloá Angélica Koehnlein \\ ORCID: https://orcid.org/0000-0001-6836-7309 \\ Federal University of Fronteira Sul, Brazil \\ E-mail: eloa.koehnlein@uffs.edu.br \\ Jucieli Weber \\ ORCID: https://orcid.org/0000-0002-5984-3593 \\ Federal University of Fronteira Sul, Brazil \\ E-mail: jucieli.weber@uffs.edu.br
}

\begin{abstract}
Objectives: Evaluate associated factors to the consumption of whole foods by adults and elderly people. Methods: Cross-sectional study carried out in a municipality in the southwest of Paraná, in which an interview was conducted, comprising 41 objective questions, in adults and the elderly. The factors associated with the consumption of whole foods were evaluated using logistic regression models. Results: The sample consisted of 388 people, predominantly of women, white people, resident in the urban area, with more than 11 years of schooling belonging to socioeconomic class B2-C1. More than a half of the participants reported consuming whole foods (54.6\%), however, $21.23 \%$ consumed them of irregular form. It was noticed that the female people (Odds Ratio: 2.24; CI 95\%=1.38-3.65), physically actives (Odds Ratio: 3.33; CI 95\%=1.89-5.87), and that had a greater knowledge about whole foods (Odds Ratio: 1.66; CI 95\%=1.01-2.74) had greater chances of consuming them. Related to the regular consumption of these foods, overweight people (Odds Ratio: 0.45; CI 95\%=0.21-0.96) presented less chances of ingestion, and the mean of communication and the health professional (Odds Ratio: 2.78; CI 95\%=1.07-7.18) presented positive influence in regular consumption of whole foods. Conclusions: There is a need to increase the consumption of whole foods in specific audiences, such as men, sedentary, lower economic class, individuals who do not have knowledge about whole foods and those who are overweight.
\end{abstract}

Keywords: Diet; Whole grains; Dietary fiber.

\begin{abstract}
Resumo
Objetivo: Avaliar fatores associados ao consumo de alimentos integrais por adultos e idosos. Métodos: Estudo transversal realizado em um município do sudoeste do Paraná, em que foi realizada uma entrevista, composta por 41 questões objetivas, em adultos e idosos. Os fatores associados ao consumo de alimentos integrais foram avaliados por meio de modelos de regressão logística. Resultados: A amostra foi composta de 388 pessoas. Mais da metade dos participantes referiram consumir alimentos integrais (54,6\%), no entanto, 21,23\% consumiam de forma irregular. Foi observado que os indivíduos do sexo feminino (Odds Ratio: 2,24; IC 95\%=1,38-3,65), ativos fisicamente (Odds Ratio: 3,33; IC 95\%=1,89-5,87), e que possuíam maior conhecimento sobre alimentos integrais (Odds Ratio:1,66; IC $95 \%=1,01-2,74)$ possuíam maiores chances de consumo dos mesmos. Com relação ao consumo regular desses alimentos, os indivíduos com excesso de peso (Odds Ratio:0,45; IC 95\%=0,21-0,96) apresentaram menores chances de ingestão, e já os meios de comunicação e os profissionais de saúde (Odds Ratio: 2,78; IC 95\%=1,07-7,18) apresentaram influência positiva no consumo regular dos alimentos integrais. Conclusão: Há necessidade de aumento do consumo de alimentos integrais em públicos alvo específicos, como homens, sedentários, menor classe econômica, indivíduos que não possuem conhecimento sobre os alimentos integrais e aqueles que se encontram acima do peso.

Palavras-chave: Dieta; Grãos integrais; Fibra alimentar.
\end{abstract}




\section{Resumen}

Meta: Evaluar factores asociados al consumo de alimentos integrales por adultos y ancianos. Métodos: Estudio transversal realizado en una ciudad del suroeste de Paraná, en el que se realizó una entrevista, compuesta por 41 preguntas objetivas, en adultos y ancianos. Los factores asociados al consumo de alimentos integrales se evaluaron mediante modelos de regresión logística. Resultados: La muestra estuvo formada por 388 personas. Más de la mitad de los participantes informaron consumir alimentos integrales (54,6\%), sin embargo, el 21,23\% los consumió de forma irregular. Se observó que las mujeres (Odds Ratio: 2,24; IC 95\% = 1,38-3,65), físicamente activas (Odds Ratio: 3,33 ; IC $95 \%=1,89-5,87$ ) y que tenían un mayor conocimiento sobre los alimentos integrales (Odds Ratio: 1,66; IC $95 \%=1.01-2.74$ ) tenían mayores posibilidades de consumirlos. En cuanto al consumo habitual de estos alimentos, las personas con sobrepeso (Odds Ratio: 0,45; IC 95\% = 0,21-0,96) tenían menores probabilidades de ingerir, al igual que los medios de comunicación y los profesionales de la salud (Odds Ratio: 2,78; IC 95\% = 1,07-7,18) mostró una influencia positiva en el consumo regular de alimentos integrales. Conclusión: Existe la necesidad de incrementar el consumo de alimentos integrales en públicos objetivo específicos, como hombres, personas sedentarias, clase económica baja, personas que no tienen conocimientos sobre alimentos integrales y personas con sobrepeso.

Palabras clave: Dieta; Granos integrals; Fibra dietética.

\section{Introduction}

Whole grain present the composition nearest of the original, and it is composed by three parts: bran, endosperm and germ (Whole Grain Council, 2017). The bran contains vitamins, minerals and fibers, presenting the function of protect the germ and the endosperm from environmental damages. The endosperm contains carbohydrates, proteins and small micronutrients amounts, offering energy to the plant. Finally, the germ contains essentials components, as micronutrients, lipids and proteins. It also contains the embryo, which is the responsible for originate a new plant (Whole Grain Council, 2017; Zhang \& Hamaker, 2017).

The whole foods consumption is related to the decrease in the mortality risk from several diseases, such as cardiovascular diseases, Diabetes Mellitus type 2, cancer, respiratory diseases, among others pathologies (Aune et al., 2016; Huang et al., 2015), as well as all-cause mortality (Chen et al., 2016; Zhang et al., 2018), demonstrating the importance of its regular eating. Meta-analysis data (Chen et al., 2016) demonstrated significant associations related to increase intake of whole grains $\left(50 \mathrm{~g} \mathrm{day}^{-1}\right)$ and reduced mortality by any cause (Relative Risk: 0.78), cardiovascular disease (DCV) (Relative Risk: 0.70). Thus, the importance of the current recommendations to increase the consumption of whole grains, improving the population health stands out.

Despite the benefits arising from the whole foods consumption and the increased demand of it in the supermarket, the Brazilian population and different parts of the world do not reach the current consumption recommendations (Albertson et al., 2016; Guiné et al., 2016; Ross et al., 2017).

Investigations on the determining factors for the consumption of whole foods are scarce. This information would be important to outline public health strategies that aim to stimulate its consumption. In addition, the standardization of definition and labeling of whole food by food industries and regulatory agencies could increase the its consumption, as people may have various worries while purchasing it, due to the labels/packaging that are not clearly arranged, making it difficult for consumers to understand.

In view of the above, this study aimed to assess the factors associated with the whole foods consumption by adults and the elderly.

\section{Methodology}

This is a quantitative, descriptive research with data collection in the field and transversal temporality. The research participants were randomly approached in the city of Francisco Beltrão - PR during the months of November 2017 to January 2018, in places with wide people circulation. 397 people were interviewed, a sample defined based on the 2010 demographic 
census, taking into consideration the total population of the city 78,943 inhabitants (Instituto Brasileiro de Geografia e Estatística, 2010). The inclusion criteria in the research were: being over 20 years of age and residents in the urban or rural area of the municipality.

For data collection, a questionnaire structured by the authors based on the literature (Pimentel \& Simões, 2012; Bendino, Popolim \& Oliveira, 2012) was applied, consisting of 41 objective questions, divided into 2 dimensions: one that included the characterization of the participants in relation to physical activity, self-reported sociodemographic, clinical, weight and height data and another including questions about knowledge and consumption characteristics of whole foods. If the individual indicated that they consumed whole foods, the interview was conducted in full, including questions about the frequency of consumption. The frequency of consumption in the last 6 months was asked in a semi-quantitative way for 5 foods (bread, pasta, biscuit, rice and whole grains) with 7 answer options, ranging from "never" to "2 or more times a day". If the participant reported not consuming whole foods, the interview ended in question 27 , which investigated the reasons for not consuming this type of food.

The socioeconomic profile was defined through the questionnaire of Brazilian Association of Research Companies (2014). Two trained researchers collected the data through a face-to-face interview. An experimental test was realized with 10 participants, to identify possible difficulties in understanding the questions and whether answers contemplated the research objectives.

This research was submitted and approved by the Committee of Moral on Research with human beings of Federal University of Fronteira Sul, CAAE: 77089417.0.0000.5564. For its performance, the participants signed the Term of Free Approval and Clarified (TCLE).

From the data of weight and height was calculated the Body Mass Index (weight/height ${ }^{2}$ ) that was classified according to World Health Organization (WHO) (1995) for adults and Lipschitz (1994) for elderly people. The level of physical activity of the participants was also classified according to WHO (2010).

The whole foods consumption was classified as regular once the participants reported consuming any type of whole food 5 or more times a week, considering an usual portion, in the last 6 months. Data were analyzed using the statistical program Stata version 13.0, using the "Pearson`s qui square! to evaluate the differences in proportions between variables, considering $\mathrm{p}<0.05$. Univariate and Multivariate Logistic Regression was performed with a 95\% confidence interval to assess the associations between the outcomes: consumption or not of whole foods and its regular/irregular consumption and the exposure variables: gender, ethnicity, home situation, age, marital status, occupation, education, social class, nutritional status, physic activity, intestine habits, pathologies, among others.

\section{Results}

From 397 individuals who answered the questionnaire, 9 were excluded due to food intolerances and/or intestine surgery, totaling the sample studied in 388 participants.

The characteristics of the sample studied are exposed in Table 1. Most of the sample was female, white, residents of the urban area, with more than 11 years of schooling and belonging to socioeconomic class B2-C1. Regarding the age of the studied population, young adults were most participants, followed by middle-aged individuals. Table 1 also displays that the whole foods consumption was reported by more than half of the participants (54.6\%), with significantly higher percentages of consumption among female (64.3\%), white ethnicity (57.8\%), > 11 years of study (64.4\%), social class A-B1 (63.1\%) and physically active people (69.4\%.). Regarding the participants' knowledge about whole foods, it was noted that those who reported consuming whole foods were those who significantly more mentioned knowing what whole foods are (63.2\%), and 
the individuals who reported not consuming whole foods were those who significantly more mentioned did not know about its composition $(70.8 \%)$.

Table 1. Social and demographic characteristics of participants, and accordingly to the consumption of whole foods and the regular consumption of them. Francisco Beltrão, 2017/2018.

\begin{tabular}{|c|c|c|c|c|c|c|}
\hline \multirow{2}{*}{$\begin{array}{l}\text { Variables }(\mathbf{n}=388) \\
\text { Gender }\end{array}$} & \multirow[b]{2}{*}{$\mathbf{n}$} & \multirow[b]{2}{*}{$\%$} & \multicolumn{2}{|c|}{$\begin{array}{l}\text { Consumption of whole foods } \\
(n=212)\end{array}$} & \multicolumn{2}{|c|}{$\begin{array}{l}\text { Regular consumption of } \\
\text { whole foods }(n=167)\end{array}$} \\
\hline & & & $\mathbf{n}$ & $\%$ & $\mathbf{n}$ & $\%$ \\
\hline Female & 241 & 62.1 & 155 & 64.3 & 127 & 81.9 \\
\hline Male & 147 & 38.9 & 57 & 38.8 & 40 & 70.2 \\
\hline \multicolumn{7}{|l|}{ Ethnicity } \\
\hline White & 348 & 89.7 & 201 & 57.8 & 159 & 79.1 \\
\hline Others & 40 & 10.3 & 11 & 27.5 & 8 & 72.7 \\
\hline \multicolumn{7}{|l|}{ Home Situation } \\
\hline Urban Area & 287 & 74.0 & 163 & 56.8 & 129 & 79.1 \\
\hline Rural Area & 101 & 26.0 & 49 & 48.2 & 38 & 77.6 \\
\hline \multicolumn{7}{|l|}{ Age group (years) } \\
\hline Young Adult (20 - 39) & 165 & 42.5 & 94 & 57.0 & 74 & 78.7 \\
\hline Middle-Age (40 - 59) & 131 & 33.8 & 70 & 53.4 & 54 & 77.1 \\
\hline Seniors $(\geq 60)$ & 92 & 23.7 & 48 & 52.2 & 39 & 81.3 \\
\hline \multicolumn{7}{|l|}{ Civil state } \\
\hline With Partner & 253 & 65.2 & 138 & 54.6 & 107 & 77.5 \\
\hline Without Partner & 135 & 34.8 & 74 & 54.8 & 60 & 81.1 \\
\hline Occupation & & & $\mathbf{n}$ & $\%$ & $\mathbf{n}$ & $\%$ \\
\hline Formal employment & 283 & 72.9 & 161 & 56.9 & 124 & 77.0 \\
\hline No formal employment & 105 & 27.1 & 51 & 48.6 & 43 & 84.3 \\
\hline \multicolumn{7}{|c|}{ Scholarity (years of study) } \\
\hline$<8$ & 87 & 22.4 & 32 & 36.8 & 23 & 71.9 \\
\hline $8-11$ & 62 & 16.0 & 26 & 41.9 & 22 & 84.6 \\
\hline$>11$ & 239 & 62.6 & 154 & 64,4 & 122 & 79.2 \\
\hline \multicolumn{7}{|l|}{ Social Class * } \\
\hline $\mathrm{A}-\mathrm{B} 1$ & 111 & 28.6 & 70 & 63.1 & 54 & 77.1 \\
\hline $\mathrm{B} 2-\mathrm{C} 1$ & 209 & 53.9 & 118 & 56.5 & 96 & 81.4 \\
\hline $\mathrm{C} 2-\mathrm{D} / \mathrm{E}$ & 68 & 17.5 & 24 & 35.3 & 17 & 70.8 \\
\hline \multicolumn{7}{|l|}{ Nutritional Status } \\
\hline Eutrophy /Underweight & 169 & 43.6 & 97 & 57.4 & 82 & 84.5 \\
\hline Overweight & 219 & 56.4 & 115 & 52.4 & 85 & 73.9 \\
\hline \multicolumn{7}{|l|}{ Physical Activity } \\
\hline Sedentary & 188 & 48.5 & 84 & 44.7 & 69 & 82.1 \\
\hline
\end{tabular}




\begin{tabular}{|c|c|c|c|c|c|c|}
\hline Not very active & 89 & 22.9 & 51 & 57.3 & 39 & 76.5 \\
\hline Active & 111 & 28.6 & 77 & 69.4 & 59 & 76.6 \\
\hline \multicolumn{7}{|l|}{ Intestinal Habit } \\
\hline Daily Evacuation & 323 & 83.3 & 42 & 64.6 & 35 & 83.3 \\
\hline $\begin{array}{l}\text { Do not evacuate every } \\
\text { day }\end{array}$ & 65 & 16.7 & 170 & 52.6 & 132 & 77.7 \\
\hline \multicolumn{7}{|l|}{ Pathologies** } \\
\hline Absence & 238 & 61.3 & 128 & 53.8 & 101 & 78.9 \\
\hline $\begin{array}{l}\text { Chronic Non } \\
\text { Transmissible Diseases }\end{array}$ & 110 & 28.4 & 64 & 58.2 & 51 & 79.7 \\
\hline $\begin{array}{l}\text { Gastrointestinal and } \\
\text { Hepatic }\end{array}$ & 13 & 3.4 & 6 & 46.2 & 5 & 83.3 \\
\hline Others & 27 & 7.0 & 14 & 51.9 & 10 & 71.4 \\
\hline Knowledge of whole & & & $\mathbf{n}$ & $\%$ & $\mathbf{n}$ & $\%$ \\
\hline \multicolumn{7}{|l|}{ foods } \\
\hline Do'nt know & 135 & 34.8 & 52 & 38.5 & 42 & 80.8 \\
\hline Knows & 253 & 65.2 & 160 & 63.2 & 125 & 78.1 \\
\hline \multicolumn{7}{|c|}{ Composition of whole food } \\
\hline Do'nt know & 120 & 30.9 & 35 & 29.2 & 28 & 80.0 \\
\hline Food Fibers & 48 & 12.4 & 34 & 70.8 & 26 & 76.5 \\
\hline $\begin{array}{l}\text { Whole Grains / No } \\
\text { Processing }\end{array}$ & 173 & 44.6 & 112 & 64.7 & 90 & 80.4 \\
\hline No Special Ingredients & 32 & 8.2 & 20 & 62.5 & 15 & 75.0 \\
\hline Others & 15 & 3.9 & 11 & 73.3 & 8 & 72.7 \\
\hline
\end{tabular}

*Social Class: A (R\$ 20,272.56), B1 (R\$ 8,695.88), B2 (R\$ 4,427.36), C1 (R\$ 2,409.01), C2 (R\$ 1,446.24), D-E (R\$ 639.78), based on the prevailing minimum wage values.

**Pathologies present in each group: DCNT: Diabetes Mellitus and Cardiovascular Diseases. Gastrointestinal and Hepatic: Diverticulitis, Gastritis, Hemorrhoids, Gastrointestinal Tract Surgery, Cholelithiasis, Hepatitis, Hepatic Steatosis). Others: Psychological problems, Respiratory, Osteoarticular, Hyper / Hypothyroidism, Cancer, etc.

Source: Authors.

Table 2 presents the association between whole foods consumption (yes or not) and sociodemographic and clinical characteristics of the sample, as well the sample's knowledge about whole foods. The variables that better explain the model and that remained the significance after the adjustment were: the female gender (Odds Ratio: 2.24), and the population considered active (Odds Ratio: 3.33) and low active (Odds Ratio: 1.94) who had higher chance to consume whole foods.

As for the knowledge about these foods, it was possible to observe that individuals who knew what whole foods were, had 1.66 higher chances of consume compared with those who did not know or never listened about these foods. It was also found that individuals who assumed that these foods should have dietary fiber, whole grains/not being processed and other components besides the mentioned had higher chances (Odds Ratio: 2.87; 2.73; and 3.37, respectively) of consumption, as for those who did not know what whole foods were. The variables that lost significance after adjusting the model were ethnicity, education and intestinal habits. However, the educational level variable was very close to being significant ( $\mathrm{p}=0.052)$ and demonstrated that individuals who have more than 11 years of study have higher chances of consumption (Odds Ratio: 1.92) in relation to those who have less than 8 years of study (Odds Ratio: 0.90). 
Table 2. Association between the sample's characteristics and the knowledge about whole foods with the consumption of them (yes or not). Francisco Beltrão, 2017/2018.

\begin{tabular}{|c|c|c|c|c|c|c|}
\hline \multicolumn{4}{|c|}{ Univariate Logistic Regression } & \multicolumn{3}{|c|}{ Regression Logistic Multivariate } \\
\hline $\begin{array}{l}\text { Explanatory } \\
\text { Variables }\end{array}$ & $\begin{array}{l}\text { Odds } \\
\text { Ratio }\end{array}$ & $\begin{array}{c}\text { Confidence } \\
\text { Interval }(95 \%)\end{array}$ & $p$-value & $\begin{array}{l}\text { Odds } \\
\text { Ratio }\end{array}$ & $\begin{array}{c}\text { Confidence } \\
\text { Interval } \\
(95 \%)\end{array}$ & $p$-value \\
\hline \multicolumn{7}{|l|}{ Gender } \\
\hline Female & 2.84 & $(1.86-4.34)$ & $<0.001$ & 2.24 & $(1.38-3.65)$ & 0.001 \\
\hline Male & 1 & & & 1 & & \\
\hline \multicolumn{7}{|l|}{ Ethnicity } \\
\hline White & 1 & & & 1 & & \\
\hline Others & 0.28 & $(0.13-0.57)$ & 0.001 & 0.49 & $(0.21-1.12)$ & 0.092 \\
\hline \multicolumn{7}{|l|}{ Home Situation } \\
\hline Urban área & 1 & & & & & \\
\hline Rural área & 0.72 & $(0.45-1.13)$ & 0.151 & & & \\
\hline \multicolumn{7}{|l|}{ Age group (years) } \\
\hline Young adult (20-39) & 1 & & & & & \\
\hline Middle-age (40-59) & 0.87 & $(0.55-1.37)$ & 0.544 & & & \\
\hline Seniors $(\geq 60)$ & 0.82 & $(0.49-1.37)$ & 0.459 & & & \\
\hline
\end{tabular}

\section{Civil State}

With Partner

Without Partner

1.01

$(0.66-1.54)$

0.96

\section{Occupation}

With formal employment

$1.4 \quad(0.89-2.19) \quad 0.144$

Without formal employment

1

Scholarity (years of study)

\begin{tabular}{lcccccc}
$8-11$ & 1 & & 1 & & & \\
$<8$ & 0.8 & $(0.41-1.57)$ & 0.525 & 0.9 & $(0.42-1.96)$ & 0.798 \\
$>11$ & $\mathbf{2 . 5 1}$ & $(\mathbf{1 . 4 2}-\mathbf{4 . 4 3})$ & $\mathbf{0 . 0 0 2}$ & 1.92 & $(0.99-3.72)$ & 0.052 \\
\hline
\end{tabular}

\section{Nutritional Status}

Eutrophy/

Underweight

Overweight

\section{Physical Activity}

Sedentary

0.82

$(0.55-1.23)$

0.338

Not very active 1

Active

$\begin{array}{cccc}1.66 & (1.00-2.76) & 0.051 & 1.94 \\ 2.8 & (1.71-4.60) & <0.001 & 3.33\end{array}$

1

2.8

$(1.71-4.60) \quad<0.001$

3.33

(1.09 - 3.44)

0.024

(1.89 - 5.87)

$<0.001$ 
Table 2. Association between the sample's characteristics and the knowledge about whole foods with the consumption of them (yes or not). Francisco Beltrão, 2017/2018. (Continues).

\begin{tabular}{|c|c|c|c|c|c|c|}
\hline $\begin{array}{l}\text { Explanatory } \\
\text { Variables }\end{array}$ & $\begin{array}{l}\text { Odds } \\
\text { Ratio }\end{array}$ & $\begin{array}{c}\text { Confidence } \\
\text { Interval }(95 \%)\end{array}$ & $p$-value & $\begin{array}{l}\text { Odds } \\
\text { Ratio }\end{array}$ & $\begin{array}{c}\text { Confidence } \\
\text { Interval } \\
(95 \%)\end{array}$ & $p$-value \\
\hline \multicolumn{7}{|l|}{ Intestinal Habit } \\
\hline Do not evacuate every day & 1 & & & 1 & & \\
\hline Daily Evacuation & 0.61 & $(0.35-1.06)$ & 0.078 & 0.77 & $(0.41-1.46)$ & 0.430 \\
\hline \multicolumn{7}{|l|}{ Pathologies* } \\
\hline Absence & 1 & & & & & \\
\hline $\begin{array}{l}\text { Chronic Non Transmissible } \\
\text { Diseases }\end{array}$ & 1.19 & $(0.76-1.89)$ & 0.443 & & & \\
\hline Gastrointestinal and Hepatic & 0.74 & $(0.24-2.26)$ & 0.593 & & & \\
\hline Others & 0.92 & $(0.42-2.05)$ & 0.849 & & & \\
\hline
\end{tabular}

\section{Social Class $* *$}

$\begin{array}{lccc}\mathrm{A}-\mathrm{B} 1 & 1 & & \\ \mathrm{~B} 2-\mathrm{C} 1 & 0.76 & (0.47-1.22) & 0.254 \\ \mathrm{C} 2-\mathrm{D} / \mathrm{E} & 0.32 & (0.17-0.60) & <0.001\end{array}$

\section{Knowledge of whole foods}

Do'nt know

Knows

\section{1}

2.75

(1.78-4.22)

$<0.001$

1

1.66

$(1.01-2.74)$

0.046

Composition of whole food

Do'nt know

Food fibers

WholeGrains / No Processing

No Special Ingredients

Others

\section{1}$$
5.9
$$$$
4.46
$$

4.05

6.68
(2.82- 12.32)

$(2.70-7.37)$

$(1.79-9.16)$

$(1.99-22.4)$
1

2.87

$(1.25$ - 6.57)

0.013

2.73

$(1.55$ - 4.81)

0.001

2.5

$(1.03$ - 6.08)

$\mathbf{0 . 0 4 3}$

3.37

$(0.91-12.39)$

0.068

*Pathologies present in each group: DCNT: Diabetes Mellitus and Cardiovascular Diseases. Gastrointestinal and Hepatic: Diverticulitis, Gastritis, Hemorrhoids, Gastrointestinal Tract Surgery, Cholelithiasis, Hepatitis, Hepatic Steatosis). Others: Psychological problems, Respiratory, Osteoarticular, Hyper / Hypothyroidism, Cancer, etc.

**Social Class: A (R\$ 20,272.56), B1 (R\$ 8,695.88), B2 (R\$ 4,427.36), C1 (R\$ 2,409.01), C2 (R\$ 1,446.24), D-E (R\$ 639.78),

based on the prevailing minimum wage values.

Source: Authors.

Table 3 presents the association between regularity (yes or no) of consumption and sociodemographic and clinical characteristics of the sample, as well the sample's knowledge about the whole foods. The variables that best explained the model and that were significantly associated with regular consumption of whole foods after adjustment were nutritional status and the reasons why respondents used whole foods. Individuals considered overweight had a lower chance (Odds Ratio: 0.45) of regular whole foods consumption compared to individuals diagnosed with eutrophy or underweight. 
With regard to the reasons why individuals use whole products, it was observed in the present study that the influence of the media/indication by a doctor and/or nutritionist had greater chances (Odds Ratio: 2.78) of regular consumption compared to those who consume only through habit or personal taste (Odds Ratio: 0.32).

Table 3. Associated factors the regular/irregular consumption of whole foods. Francisco Beltrão, 2017/2018.

\section{Univariate Logistic Regression}

\section{Explanatory}

Variables

Odds

Ratio

\section{Confidence}

Interval (95\%)
Logistic Regression Multivariate

$\begin{array}{cccc}p \text {-value } & \text { Odds } & \text { Confidence } & p \text {-value } \\ & \text { Ratio } & \text { Interval }(95 \%) & \end{array}$

\section{Gender}

Female

1.93

$(0.96-3.88)$

0.066

1.03

$(0.45-2.34)$

0.947

Male

1

1

\section{Ethnicity}

White

Others

\section{1}

\section{Home Situation}

Urban área

0.7

$(0.18-2.77)$

0.616

Rural área

0.91

$(0.42-1.97)$

0.811

Age group (years)

Young Adult (20-39)

Middle-Age (40-59)

1

Seniors (> de 60)

0.91

$(0.43-1.92)$

0.809

\section{Civil State}

With Partner

Without Partner

1.24

$(0.61-2.51)$

0.548

\section{Occupation}

With formal employment

0.62

$(0.27-1.44)$

0.27

Without formal employment

Scholarity (years of study)

$<8$

8-11

0.46

$>11$

1

$(0.12-1.73)$

0.253

\section{Nutritional Status}

Eutrophy/

Underweight

1

$(0.22-2.15)$

0.527

Overweight

0.52

$(0.26-1.03)$

0.062

0.45

$(0.21-0.96)$ 
Table 3. Associated factors the regular/irregular consumption of whole foods. Francisco Beltrão, 2017/2018. (Continues).

\begin{tabular}{lccccc}
\hline & Univariate Logistic Regression & & Logistic Regression Multivariate \\
\hline $\begin{array}{l}\text { Explanatory } \\
\text { Variables }\end{array}$ & $\begin{array}{c}\text { Odds } \\
\text { Ratio }\end{array}$ & $\begin{array}{c}\text { Confidence } \\
\text { Interval }(95 \%)\end{array}$ & $\boldsymbol{p}$-value & $\begin{array}{c}\text { Odds } \\
\text { Ratio }\end{array}$ & $\begin{array}{c}\text { Confidence } \\
\text { Interval (95\%) }\end{array}$ \\
\hline Physical Activity & 1 & & & \\
Sedentary & 0.706 & $(0.30-1.66)$ & 0.426 & \\
Not very active & 0.71 & $(0.33-1.54)$ & 0.387 & \\
Active & & &
\end{tabular}

\section{Intestinal Habit}

Do not evacuate every day
Daily Evacuation
0.69
$(0.28-1.69)$
0.422

\section{Pathologies*}

Absence 1

Chronic Non

Transmissible Diseases

$\begin{array}{lll}1.05 & (0.50-2.20) & 0.9\end{array}$

Gastrointestinal and

Hepatic

$1.34 \quad(0.15-11.93) \quad 0.795$

Others

0.67

$(0.19-2.30)$

0.522

Social Class**

$\begin{array}{lccc}\mathrm{A}-\mathrm{B} 1 & 1 & & \\ \mathrm{~B} 2-\mathrm{C} 1 & 1.29 & (0.63-2.67) & 0.487 \\ \mathrm{C} 2-\mathrm{D} / \mathrm{E} & 0.72 & (0.25-2.04) & 0.536\end{array}$

Knowledge of whole foods

Do'nt know

Knows

0.85

$(0.39-1.86)$

0.686

\section{Composition of whole foods}

Do'n tknow

Food fibers

1

Whole Grains / No

Processing

$\begin{array}{lll}0.81 & (0.26-2.56) & 0.723\end{array}$

$1.02 \quad(0.39-2.64) \quad 0.963$

No Special Ingredients

$\begin{array}{lll}0.75 & (0.20-2.77) & 0.666 \\ 0.67 & (0.14-3.18) & 0.611\end{array}$

Others

0.67

$(0.14-3.18)$

0.611

Time of inclusion of whole

\section{foods in the diet}

$<1$ year

2 to 3 years 1

$>4$ years

$\begin{array}{lll}1.04 & (0.42-2.58) & 0.935 \\ 0.79 & (0.35-1.78) & 0.568\end{array}$


Table 3. Associated factors the regular/irregular consumption of whole foods. Francisco Beltrão, 2017/2018. (Continues).

\begin{tabular}{|c|c|c|c|c|c|c|}
\hline \multicolumn{4}{|c|}{ Univariate Logistic Regression } & \multicolumn{3}{|c|}{ Logistic Regression Multivariate } \\
\hline $\begin{array}{l}\text { Explanatory } \\
\text { Variables }\end{array}$ & & $\begin{array}{l}\text { Confidence } \\
\text { iterval }(95 \%)\end{array}$ & $p$-value & $\begin{array}{l}\text { Odds } \\
\text { Ratio }\end{array}$ & $\begin{array}{c}\text { Confidence } \\
\text { Interval }(95 \%)\end{array}$ & $p$-value \\
\hline \multicolumn{7}{|l|}{ Reason for using whole foods } \\
\hline Healthier / Lose Weight & 1 & & & 1 & & \\
\hline Habit and For Liking & 0.31 & $(0.12-0.81)$ & 0.017 & 0.32 & $(0.12-0.90)$ & $\mathbf{0 . 0 3 1}$ \\
\hline $\begin{array}{l}\text { Influence of the media or by medical / } \\
\text { nutritionist indication }\end{array}$ & 2.37 & $(0.97-5.76)$ & 0.058 & 2.78 & $(1.07-7.18)$ & $\mathbf{0 . 0 3 5}$ \\
\hline \multicolumn{7}{|c|}{ How to obtain information on whole foods } \\
\hline Never repaired the information & 1 & & & 1 & & \\
\hline Media & 2.88 & $(0.64-13.04)$ & 0.17 & 2.47 & $(0.43-14.20)$ & 0.311 \\
\hline Health professionals & 2.9 & $(0.54-15.55)$ & 0.214 & 1.99 & $(0.30-13.27)$ & 0.475 \\
\hline Food label & 0.9 & $(0.13-6.08)$ & 0.914 & 0.89 & $(0.10-7.60)$ & 0.919 \\
\hline Others & 1.55 & $(0.32-7.52)$ & 0.586 & 1.39 & $(0.22-8.46)$ & 0.719 \\
\hline
\end{tabular}

Understanding of information about whole foods

$\begin{array}{lccc}\text { Never repaired the information } & 1 & & \\ \text { Easy understanding } & 1.97 & (0.73-5.29) & 0.179 \\ \text { Moderate/Difficult understanding } & 1.1 & (0.38-3.17) & 0.863\end{array}$

\section{Cost}

Accessible/Very accessible

Moderate

1.01

$(0.42-2.43)$

0.978

High/Very High

1.32

$(0.57-3.09)$

0.516

\section{Items observed for purchase decision of whole food}

Do not watch any item

1

Brand/Price/Indication of relatives and health professional;

Label (list of ingredients, nutritional information, the word "whole food", etc).

$\begin{array}{lllll}2.16 & (0.94-4.99) & 0.07 & 1.77 & (0.71-4.44) \\ 2.55 & (1.08-6.06) & 0.033 & 2.15 & (0.82-5.65)\end{array}$

\section{1}

$(0.82-5.65)$

\footnotetext{
*Pathologies present in each group: DCNT: Diabetes Mellitus and Cardiovascular Diseases. Gastrointestinal and Hepatic: Diverticulitis, Gastritis, Hemorrhoids, Gastrointestinal Tract Surgery, Cholelithiasis, Hepatitis, Hepatic Steatosis). Others: Psychological problems, Respiratory, Osteoarticular, Hyper / Hypothyroidism, Cancer, etc.

**Social Class: A (R\$ 20,272.56), B1 (R \$ 8,695.88), B2 (R\$ 4,427.36), C1 (R\$ 2,409.01), C2 (R\$ 1,446.24), D-E (R\$ 639.78), based on the prevailing minimum wage values.

Source: Authors.
}

\section{Discussion}

In this study developed in the city of Francisco Beltrão, it was found that $54.6 \%$ of the participants mentioned consuming whole food; however, $1 / 5$ of them (21.2\%) mentioned irregular frequency consumption. We also found a significant association between being female, physically active, and to have knowledge about whole foods with higher chance of consumption of these foods. Regarding the regular consumption of whole foods we observed that is inversed associated to overweight and positively associated to the influence of the media or because indication of a professional health. 
Previous studies have also found low consumption frequency of whole foods. In Malaysia, a study with adult university students found that $51 \%$ reported these foods consumption in the last 3 months, with only $13 \%$ having daily consumption (Subramanian et al., 2019). In Portugal, an assessment of the attitudes of 382 adults towards dietary fibers found that the frequency of whole grains consumption by the participants was low, being once every two days of the week (Guiné et al., 2016). Mann et al. (2015) suggested that the increase of whole grains consumption must be encouraged, once the small portions consumption can cause an improvement in the diet, such as greater intake of fibers, magnesium and iron and less intake of sodium, therefore, improvement in the health of individuals. Parker et al. (2013) noticed that a consumption of more than two portions of whole grains/day by women between 50 and 79 years resulted in a $43 \%$ decrease in the risk of incident diabetes, when compared to women who did not have this daily consumption.

Attitudes related to the whole grains consumption are positively correlated with individuals' knowledge about it (Koo et al., 2018). Data similar to those found in this study were found in the study of Guiné et al. (2016) in which it was identified that female gender (Mean: $3.63 \pm 3.58$ meals/week) and people with higher education (Mean $\pm \mathrm{DP}: 3.32 \pm 3.47 \mathrm{meals} / \mathrm{week}$ ) presented higher consumption of meals containing whole foods in the week, in comparison to the male gender (Mean $\pm \mathrm{DP}$ : 2.30 \pm 2.87 meals/week) and individuals who had less education (Mean \pm DP: $2.26 \pm 2.86$ meals/week).

Similar result was noticed by Parker et al. (2013) that evaluated the whole grains consumption by women and its association with type 2 diabetes mellitus. The authors identified that women who presented a higher whole grains consumption (>2 portions/day) were more active in relation to physical exercises (16.5 hours/week) compared to 11 hours of physical activity/week for those who did not consume any portion of whole foods. They also presented higher education (university degree or higher) and were less likely to be current smokers, compared to those who did not consume any portion of whole grain on the day. Another study (Huang et al., 2015) also found that the levels of physical activity considered moderate and vigorous were higher in individuals who presented higher whole grains consumption in comparison to those who obtained lower consumption.

Vaidean et al. (2017) performed a study with middle-aged adults (between 45 and 64 years old) from southeastern Poland on the frequency of whole grains consumption appropriate and the levels of serum lipids associated with the intake of statin. The authors noticed, among statin users and non-statin users, the association between appropriate whole grains consumption and several variables, as being female, having higher education, living in an urban area, being more physically active, among others. The authors also noticed that rural, males and individuals with less education presented lower whole grains consumption (Vaidean et al., 2015). Although not statistically significant, in the present study, individuals who lived in the rural area also presented lower whole products consumption compared to residents in the urban area.

A study carried out in the United States of America (USA) on the consumption of fibers and whole grains in all ages found low total consumption of both, not reaching the current recommendations (Mc Gill, Fulgoni \& Devareddy, 2015). The average fibers consumption of adults was $16.1 \pm 0.2 \mathrm{~g} / \mathrm{day}$, whereas the average whole grains consumption by adults was 17.29 $\pm 0.56 \mathrm{~g} /$ day and for adults aged $>51$ years, was $24.38 \pm 0.56 \mathrm{~g} /$ day.

As noticed, individuals who presented greater knowledge about whole foods, consequently presented higher chance of consuming it. A study that corroborates with this finding was developed by Koo et al. (2018), who observed positive and meaningful correlation between the students' knowledge about whole grains with attitudes $(r=0.337$; $\mathrm{p}<0.01)$ and practices $(\mathrm{r}$ $=0.190 ; \mathrm{p}<0.01)$ regarding to its consumption. An assessment of the Croatian population over the age of 18 years found a weak association between the consumption of foods rich in fiber and the knowledge about it (Ljubicic et al., 2017). Among the different barriers to the whole foods consumption, we can mention the inability to identify whole foods (Barret, Foster \& Beck, 2020). 
The research developed by Koo et al. (2018), in which the authors evaluated the knowledge, attitudes and students practices in relation to whole grains, identified that eutrophic individuals had practices related to higher whole grains consumption (score:29), in relation to the punctuation obtained through a questionnaire, than by obese or overweight people (score: 27.25), being these data similar to the present study.

The variable that lost its significance after adjusting the model was the question about the items that the individuals observed to decide which whole food buy, despite being non-significant at the 5\% confidence level (0.119), presented interesting data, being that individuals who observed the label information are more likely to have regular whole foods consumption compared to those who do not observe any item. Christoph et al. (2018) noticed that the use of nutritional label information by young adults (25 to 36 years old) in Minnesota, was associated with higher intake of food considered healthy, data similar to the present study.

In the study developed by Koo et al. (2018) it was observed that although the students reported not liking whole grains, 39.3\% still consumed it, which differs from the data found in this research, since the people who signaled that they did not like whole grains, did not consumed.

Aversion to the organoleptic properties (taste, texture, appearance and smell) of whole foods is one of the main difficulties for increasing the whole grains consumption, which is associated with the price of these foods, greater time for preparation and cooking (Barret, Foster \& Beck, 2020). The issue of habit as a barrier to the consumption of these foods was also found in the literature, as well as family preference and availability (Neo \& Brownlee, 2017).

It was noted in the study that the usual question was the most prevalent among the reasons for not consuming whole foods. Therefore, strategies must be used to change this scenario, in order to obtain increased consumption of these foods. A strategy that could be listed in view of the results found would be greater dissemination in the media about whole foods and its numerous benefits.

Seal et al. (2016) suggest that to achieve an increase in the consumption of whole grains, it is necessary that health and industry bodies establish a standard to correctly define whole foods, making consumption to be optimized. In Brazil, until early 2021, there was no legislation that established the definition of whole foods, nor that it determined mandatory standards of proportionality of whole grains in foods to be considered whole foods. On April 15, 2021, resolution RDC 493 was published, which provides for the composition and labeling requirements of foods containing cereals for classification and identification as whole and to highlight the presence of whole ingredients. This document established the definition of integral ingredient, which would be that coming from whole cereals or their derivatives, with the typical parts and proportions that occur in intact caryopsis: endosperm, starch, bran and germ. In addition, the legislation defined that for a food containing cereals to be classified as whole, it must contain at least $30 \%$ of whole ingredients in its composition; and quantity of whole ingredients greater than quantity of refined ingredients. A period of 12 to 24 months was established, depending on the type of food, for adjustments according to the resolution.

Another alternative that has been studied would be the addition of cereal fibers to refined products, such as resistant starch or processed bran to increase the fiber content without changing the texture or flavor of the food. However, there is a lack of studies comparing the potential effect of this addition to health compared to the effects of eating whole grains (Barret, Foster \& Beck, 2020).

\section{Conclusion}

There was a low whole food consumption in the studied population, being the factors habit and the food tasty, the principal reasons pointed out for not consuming it. The factor associated with higher chances of consuming whole foods were 
belonging to the female gender, being physically active, from a higher socioeconomic class, and having greater knowledge about whole foods. Regarding to the regular consumption of these foods, overweight individuals were less likely to consume.

We highlight the necessity for further elucidation of these factors in order to increase the whole foods consumption in specific target audience, as men, sedentary, lower economic class, individuals without knowledge about whole foods and overweight individuals.

\section{References}

ANVISA. Agência Nacional de Vigilância Sanitária. Resolução da diretoria colegiada- RDC nº 493, de 15 de abril de 2021 . www.anvisa.gov.br/legis

Albertson, A. M., Reicks, M., Joshi, N., Gugger, C. K. (2016). Whole grain consumption trends and associations with body weight measures in the United States: results from the cross sectional National Health and Nutrition Examination Survey 2001-2012. Nutr J. 15(8), 1-14. http://dx.doi.org/10.1186/s12937016-0126-4

Associação Brasileira de Estudos Populacionais (ABEP) (2014). Classificação brasileira do padrão de econômico. Brasil. http://www.abep.org/criterio-brasil

Aune, D., Keum, N., Giovannucci, E., Fadnes, L. T., Boffetta, P., Greenwood, D. C., et al. (2016). Whole grain consumption and risk of cardiovascular disease, cancer, and all cause and cause specific mortality: systematic review and dose-response meta-analysis of prospective studies. BMJ. 353 , i2716. http://dx.doi.org/10.1136/bmj.i2716

Barrett, E. M., Foster, S. I., Beck, E. J. (2020). Whole grain and high-fibre grain foods: How do knowledge, perceptions and attitudes affect food choice? Appetite 149, 104630. http://dx.doi.org/10.1016/j.appet.2020.104630

Bendino, N. I., Popolim, W. O., Oliveira, C. R. A. (2012). Avaliação do conhecimento e dificuldades de consumidores frequentadores de supermercado convencional em relação à rotulagem de alimentos e informação nutricional. J Health Sci Inst. 30(3), 261-265.

Chen, G. C., Tong, X., Xu, J. Y., Han, S. F., Wan, Z. X, Qin, J. B., et al. (2016). Whole-grain intake and total, cardiovascular, and cancer mortality: a systematic review and meta-analysis of prospective studies. Am J Clin Nutr. 104(1), 64-72. http://dx.doi.org/10.3945/ajcn.115.122432

Christoph, M. J., Larson, N., Laska, M. N., Neumark-Sztainer, D. (2018). Nutrition Facts Panels: Who Uses Them, What Do They Use, and How Does Use Relate to Dietary Intake? J Acad Nutr Dietetics. 118(2), 217-228. http://dx.doi.org/10.1016/j.jand.2017.10.014

Guiné, R., Ferreira, M., Correia, P., Duarte, J. (2016). Attitudes of the Portuguese population regarding dietary fiber. Millenium 2(1), 49-62. http://dx.doi.org/10.29352/mill0201.04.00037

Huang, T., Xu, M., Lee, A., Cho, S., Qi, L. (2015). Consumption of whole grains and cereal fiber and total and cause-specific mortality: prospective analysis of 367,442 individuals. BMC Medicine. 13(59), 1-9. http://dx.doi.org/10.1186/s12916-015-0294-7

Instituto Brasileiro de Geografia e Estatística. Censo Demográfico 2010. IBGE. https://cidades.ibge.gov.br

Koo, H. C., Lee, C. L., Nur Hidayah, A. S., Nurain Hazwani, A. R. (2018). Knowledge, attitudes and practices of schoolchildren toward whole grains and nutritional outcomes in Malaysia. Appetite. 123(1), 256-263. http://dx.doi.org/10.1016/j.appet.2018.01.002

Lipschitz, D. A. (1994). Screening for nutritional status in the elderly. Prim Care. 21(1), 55-67.

Ljubicic, M., Saric, M. M., Rumbak, I., Baric, I.C., Komes, D., Satalic, Z., et al. (2017). Knowledge about dietary fibre and its health benefits: A crosssectional survey of 2536 residents from across Croatia. Medical Hypotheses. 105, 25-31. http://dx.doi.org/10.1016/j.mehy.2017.06.019

Mann, K. D., Pearce, M. S., McKevith, B., Thielecke, F., Seal, C. J. (2015). Whole grain intake and its association with intakes of other foods, nutrients and markers of health in the National Diet and Nutrition Survey rolling programme 2008-11. British J Nutr. 113(10), 1595-1602. http://dx.doi.org/10.1017/S0007114515000525

Mc Gill, C. R., Fulgoni, V. L., Devareddy, L. (2015). Ten-Year Trends in Fiber and Whole Grain Intakes and Food Sources for the United States Population: National Health and Nutrition Examination Survey 2001-2010. Nutrients. 7(2), 1119-30. http://dx.doi.org/10.3390/nu7021119

Neo, J. E, Brownlee, I. A. (2017). Whole-grain food acceptance in young Singaporean adults. Nutrients. 9(4), 1-11. http://dx.doi.org/10.3390/nu9040371

Parker, E. D., Liu, S., Van Horn, L., Tinker, L. F., Shikany, J. M., Eaton, C. B., et al. (2013). The association of whole grain consumption with incident type 2 diabetes: the Women's Health Initiative Observational Study. Annals Epid. 23(6), 321-27. http://dx.doi.org/10.1016/j.annepidem.2013.03.010

Pimentel, T. C. \& Simões, G. S. (2012). Percepção dos consumidores em relação às fibras alimentares e seus produtos. Rev Bras Pesq Alimentos 3(1), 11-18. Ross, A. B., van der Kamp, J.W., King, R., Lê, K.A., Mejborn, H., Seal, C.J., et al. (2017). Perspective: A Definition for Whole-Grain Food Products Recommendations from the Health grain Forum. Adv Nutr. 8(4), 525-31. http://dx.doi.org/10.3945/an.116.014001

Seal, C. J., Nugent, A. P., Tee, E. S., Thielecke, F. (2016). Whole-grain dietary recommendations: the need for a unified global approach. Brit J Nutr. 115(11), 2031-38. http://dx.doi.org/10.1017/S0007114516001161

Subramanian, S., Patil, S. S., Ponnusamy, S., Hasamnis, A. A., Loh, K. Y., Santosh, N. (2019). Whole-grain consumption and its determinants in Malaysian medical students: A cross-sectional study. Indian J Pub Health. 63(3), 220-6. http://dx.doi.org/10.4103/ijph.IJPH_278_18 
Research, Society and Development, v. 10, n. 12, e84101219843, 2021

(CC BY 4.0) | ISSN 2525-3409 | DOI: http://dx.doi.org/10.33448/rsd-v10i12.19843

Vaidean, G. D., Manczuk, M., Vansal, S. S., Griffith, J. (2017). The cholesterol-lowering effect of statins is potentiated by whole grains intake. The Polish Norwegian Study (PONS). Europ J Intern Medicine. 50(1), 47-51. http://dx.doi.org/10.1016/j.ejim.2017.11.005

Whole grain council (2017). What's a whole grain? A refined grain? Boston: WGC. http://wholegrainscouncil.org/whole-grains-101/whats-whole-grainrefined-grain

World Health Organization (1995). Physical status: the use of and interpretation of anthropometry. Report of a WHO Expert Committee. https://www.who.int/childgrowth/publications/physical_status/en/

World Health Organization (2010). Global recomendations on physical activity for health. https://apps.who.int/iris/bitstream/handle/ 10665/44399/9789241599979_eng.pdf;jsessionid=7F241225D77B55E2D1CF162F3CD5C86D?sequence=1

Zhang, G. \& Hamaker, B. R. (2017). The nutritional property of endosperm starch and its contribution to the health benefits of whole grain foods. Crit Rev Food Sci Nutr. 57(18), 3807-17. http://dx.doi.org/10.1080/10408398.2015.1130685

Zhang, B., Zhao, Q., Guo, W., Bao, W., Wang, X. (2018). Association of whole grain intake with all-cause, cardiovascular, and cancer mortality: a systematic review and dose-response meta-analysis from prospective cohort studies. Eur J Clin Nutr. 72(1), 57-65. http://dx.doi.org/10.1038/ejen.2017.149 